\title{
Non-adiabatic stripping of a cavity field from electrons in the deep-strong coupling regime
}

\author{
M. Halbhuber ${ }^{1,4}$, J. Mornhinweg ${ }^{1,4}$, V. Zeller ${ }^{1}$, C. Ciuti ${ }^{2}$, D. Bougeard', R. Huber $\oplus^{1 凶}$ \\ and C. Lange $\mathbb{1}^{1,3 凶}$
}

\begin{abstract}
Atomically strong light pulses can drive sub-optical-cycle dynamics. When the Rabi frequency-the rate of energy exchange between light and matter-exceeds the optical carrier frequency, fascinating non-perturbative strong-field phenomena emerge, such as high-harmonic generation and lightwave transport. Here, we explore a related novel subcycle regime of ultimately strong light-matter interaction without a coherent driving field. We use the vacuum fluctuations of nanoantennas to drive cyclotron resonances of two-dimensional electron gases to vacuum Rabi frequencies exceeding the carrier frequency. Femtosecond photoactivation of a switch element inside the cavity disrupts this 'deep-strong coupling' more than an order of magnitude faster than the oscillation cycle of light. The abrupt modification of the vacuum ground state causes spectrally broadband polarization oscillations confirmed by our quantum model. In the future, this subcycle shaping of hybrid quantum states may trigger cavity-induced quantum chemistry, vacuum-modified transport or cavity-controlled superconductivity, opening new scenarios for non-adiabatic quantum optics.
\end{abstract}

S trong-field light-matter interaction is characterized by polarization dynamics occurring faster than an oscillation cycle of light ${ }^{1-5}$. Extremely strong electric fields have facilitated carrier-wave Rabi flopping ${ }^{2}$ and non-perturbative, lightwave-driven electron dynamics including high-harmonic generation ${ }^{1,3-5}$ and subcycle transport ${ }^{3-7}$. Subcycle temporal resolution has been crucial in understanding the underlying physics. An opposing limit has been explored in optical microcavities, where novel states of matter can be created by dressing electronic excitations with virtual photons. When the corresponding vacuum Rabi frequency, $\Omega_{\mathrm{R}}$ (the energy exchange rate between a cavity mode and an electronic resonance) assumes a sizeable fraction of the carrier frequency, $\omega_{0}$, intriguing cavity quantum electrodynamic effects ${ }^{8,9}$, such as the vacuum Bloch-Siegert shift ${ }^{10}$, modified electronic transport ${ }^{11,12}$ and cavity-controlled chemical reactions ${ }^{13,14}$, arise. Ongoing efforts to increase the coupling strength ${ }^{8-11,15-21}$ have recently opened up the regime of $\Omega_{\mathrm{R}} / \omega_{0}>1$ (refs. ${ }^{19,21}$ ). Such vacua in the deep-strong coupling regime $\mathrm{e}^{19,21}$ can host exotic quantum effects ${ }^{19,22}$, including giant Lamb shifts ${ }^{21}$, a sizeable population of virtual photons ${ }^{23}$ or cavity-mediated superconductivity ${ }^{24,25}$.

While these studies have focused on equilibrium aspects, subcycle changes of $\Omega_{\mathrm{R}}$ have been predicted to open up a new field of non-adiabatic cavity light-matter interaction. For instance, a switching-induced transfer of energy into the quantum vacuum ${ }^{26,27}$ is predicted to release the virtual photon population of the correlated ground state, similar to the Unruh-Hawking radiation of black holes $^{28}$. Abrupt undressing of the matter excitation also promises new ways of investigating the interaction dynamics of vacuum fields with electron transport ${ }^{11,12}$, Cooper pair bonding ${ }^{24,25}$ or molecular reactions ${ }^{13,14}$. Yet, thus far, non-adiabatic switching has been limited to $\Omega_{\mathrm{R}} / \omega_{0} \leq 0.1$ (ref. ${ }^{16}$ ). Subcycle control of deep-strong coupling comes with stricter yet unresolved challenges of integrating electronic excitations with giant dipole moments and optical switching functionalities into extremely subwavelength cavity mode volumes.
Here we demonstrate an abrupt undressing of light-matter coupled electrons in the deep-strong coupling regime with $\Omega_{\mathrm{R}} / \omega_{0}=1.3$ within $0.05 \times T_{0}$, where $T_{0}=2 \pi / \omega_{0}$ is the oscillation period of light, by stripping off the cavity field from an electronic excitation. To this end, femtosecond photodoping of a switch structure deactivates the fundamental cavity mode and reduces the amplitude of the resonator field interacting with an electronic resonance by more than an order of magnitude. The strongly subcycle collapse of $\Omega_{\mathrm{R}}$ and the polariton modes is characterized by pronounced high-frequency polarization oscillations evolving faster by an order of magnitude than a single oscillation cycle of the lower polariton. Our dynamical quantum model links these signatures to an extremely non-adiabatic interruption of the energy exchange of light-matter coupled modes in the deep-strong coupling regime, which opens a new dimension for future investigations of the dynamics of strongly squeezed quantum vacua.

\section{Design of switchable vacua in the deep-strong coupling regime}

Our structures couple the electric field of a metallic terahertz $(\mathrm{THz})$ resonator to the cyclotron resonance (CR) of two-dimensional, high-mobility electron gases in GaAs quantum wells (QWs) ${ }^{10,11,18-20}$ separated by AlGaAs barriers (Fig. 1a and also see Methods). At a temperature of $10 \mathrm{~K}$, a variable magnetic bias oriented perpendicularly to the QW plane leads to Landau quantization with a tunable $\mathrm{CR}$ frequency, $\nu_{c}$. The resonators (Fig. 1a) consist of an inductive (L) outer ring and a capacitive $(\mathrm{C})$ gap element, which determine the resonance frequencies and spatial shapes of the fundamental LC mode and all other optical modes. The design of our structures was guided by finite-element frequency-domain calculations ${ }^{19}$ (see Methods). In particular, the fundamental LC mode is tailored to concentrate the electric near-field within the central gap region (Fig. 1b-d), optimize its overlap with the polarization field of the CR, and thereby maximize the strength of light-matter interaction ${ }^{19}$.

'Department of Physics, University of Regensburg, Regensburg, Germany. ${ }^{2}$ Université de Paris, Laboratoire Matériaux et Phénomènes Quantiques, CNRS, Paris, France. ${ }^{3}$ Present address: Fakultät Physik, Technische Universität Dortmund, Dortmund, Germany. ${ }^{4}$ These authors contributed equally: M. Halbhuber, J.Mornhinweg.『e-mail: rupert.huber@ur.de; christoph.lange@ur.de 
a

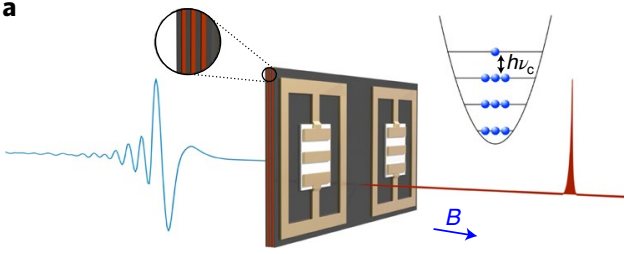

b

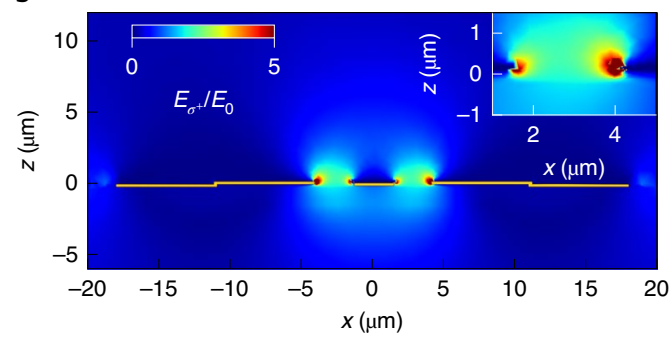

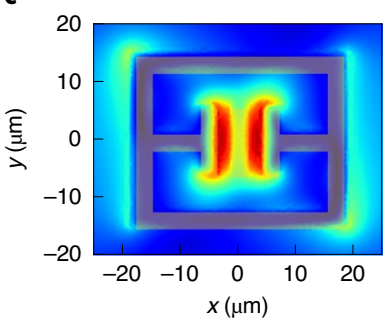

d

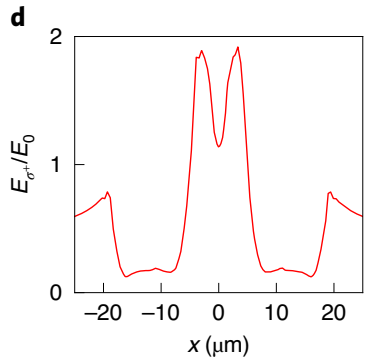

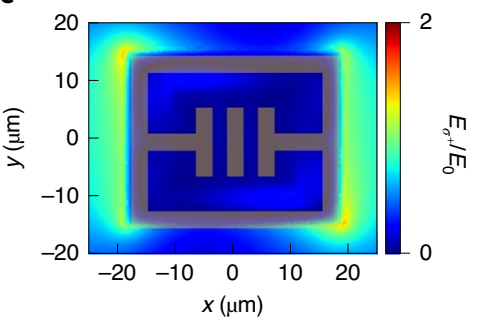

f

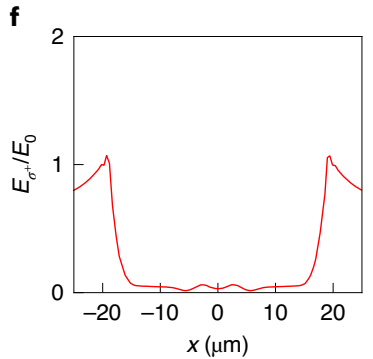

Fig. 1 | Resonator scheme for switchable deep-strong light-matter coupling. a, The light fields of THz resonators (gold shapes) are coupled to the CRs of two-dimensional electron gases (red layers, also see magnification) kept at a temperature of $10 \mathrm{~K}$, biased by a magnetic field $B$ (blue arrow), and interrogated by $\mathrm{THz}$ transients (blue waveform). A switch element (white patch) placed in the area of maximum field enhancement is photoexcited by a near-infrared femtosecond pulse (red shape) to disrupt light-matter coupling. Inset: schematic of Landau states. b, Calculated enhancement of the right-circularly polarized near-field component $E_{\sigma^{+}} / E_{0}$, to which the CR selectively couples, where $E_{0}$ is the far-field amplitude. The data are calculated at the resonance frequency of the LP mode. Inset: zoom-in of the gap region. c, Near-field enhancement in the QW plane, $200 \mathrm{~nm}$ below the surface. $\mathbf{d}$, Near-field enhancement in the QW plane along $y=0$ without photoexcitation. $\mathbf{e}$, The same as in $\mathbf{c}$ but with photoexcited charge carriers at a concentration of $\rho_{\text {switch }}=$ $3.5 \times 10^{18} \mathrm{~cm}^{-3}$ in the switching patch, squeezing the LC mode out of the gap region. $\mathbf{f}$, Corresponding near-field enhancement in the $\mathrm{QW}$ plane along $y=0$.

Correspondingly, changes of the near field within the region of maximum overlap-the QW plane-strongly influence $\Omega_{\mathrm{R}}$.

We exploit this idea to deactivate light-matter coupling all-optically. To this end, we place an $\mathrm{In}_{0.55} \mathrm{Ga}_{0.45}$ As patch between the capacitive resonator region ${ }^{29}$ and the QWs (Fig. 1a, white patch, and also see Methods). The alloy features a bandgap of $0.73 \mathrm{eV}$, which enables the selective excitation of charge carriers by femtosecond switching pulses with a photon energy of $1.03 \mathrm{eV}$. As a result, a quasi-metallic $\mathrm{THz}$ response can be induced exclusively in the patch, causing a subcycle cut-off ${ }^{30}$ of the electric near-field. Numerical calculations show that a photoexcited plasma with an electron-hole pair density of $\rho_{\text {switch }}=3.5 \times 10^{18} \mathrm{~cm}^{-3}$ efficiently expels the near field out of the resonator gap and, thus, deactivates the LC mode (Fig. 1e,f and Extended Data Fig. 1). In the gap region, the Landau electrons in the QWs are stripped of the vacuum field that drops to less than $5 \%$ of its value without excitation (Fig. 1d,f), leading to a collapse of the coupling strength.

We experimentally test our concept with two structures consisting of 3 QWs and 6 QWs, respectively, each doped with a concentration of $1.75 \times 10^{12} \mathrm{~cm}^{-2}$ electrons per QW and covered with an array of resonators designed for a frequency of the LC mode of $\nu_{\mathrm{LC}}=\omega_{\mathrm{LC}} / 2 \pi=0.85 \mathrm{THz}(3-\mathrm{QW}$ structure) and $0.5 \mathrm{THz}(6-\mathrm{QW}$ structure). Linearly polarized, single-cycle $\mathrm{THz}$ transients (Fig. 1a, blue waveform) are focused through the structures and detected electro-optically (see Methods and Extended Data Fig. 2). Lightmatter coupling leads to dressing of electrons by the vacuum field, which forms new eigenstates called cavity polaritons. In the transmission spectra, these resonances manifest by characteristic minima, whose frequencies depend on the detuning between the LC mode and the CR. We systematically control $\nu_{c}$ by varying the magnetic bias field while recording the transmission spectra of the structures.

\section{Deep-strong coupling and its optical deactivation}

The data for the 3-QW sample (Fig. 2a) clearly exhibit a lower polariton (LP) branch that emerges from the CR at low frequencies, while the upper polariton (UP) resonance is observed at $1.35 \mathrm{THz}$ for $\nu_{\mathrm{c}}=0 \mathrm{THz}$. As $\nu_{\mathrm{c}}$ increases, the polariton frequencies increase with opposite curvatures, leading to the characteristic anti-crossing shape. Owing to the extremely strong light-matter coupling, even the minimum spectral separation at the anti-crossing point $\left(\nu_{\mathrm{c}}=\right.$ $\nu_{\mathrm{LC}}$ ) still exceeds the carrier frequency of light; here the LP and UP frequencies are $\nu_{\mathrm{LP}}=0.48 \mathrm{THz}$ and $\nu_{\mathrm{UP}}=1.49 \mathrm{THz}$. Under these conditions, the vacuum ground state is populated by 0.1 virtual photons resulting from the sizeable contribution of anti-resonant light-matter exchange. An additional coupled resonance occurs approximately $0.2 \mathrm{THz}$ below the UP branch. Terahertz absorption by the bare CR in uncoupled areas between resonator structures explains the prominent transmission minimum at $\nu_{\mathrm{c}}$ (Fig. 2a, red diagonal region). We determine a coupling strength of $\Omega_{\mathrm{R}} / \omega_{\mathrm{LC}}=$ 0.57 by diagonalizing the corresponding Hamiltonian and adjusting the parameters for best agreement with the experimental spectra (see Methods). Applying the same procedure for the 6-QW structure yields a coupling strength of as much as $\Omega_{\mathrm{R}} / \omega_{\mathrm{LC}}=1.3$ (Fig. $2 \mathrm{c}$ ), leading to a ground-state population of 0.32 virtual photon pairs and amplitudes of the counter-rotating field components ${ }^{23}$ that exceed those of the resonant fields.

Next, we excite the switching patch by a near-infrared pump pulse (Fig. 1a, red pulse) with a duration of $70 \mathrm{fs}$, set to arrive at the sample 2 ps before the maximum of the $\mathrm{THz}$ pulse. The switched structure exhibits a fundamentally different transmission spectrum (Fig. 2b) characterized by the complete absence of both polariton resonances. This behaviour evidences a full collapse of $\Omega_{\mathrm{R}}$. At the same time, other spectral features, such as the bare CR, remain mostly unaffected, demonstrating the minimally invasive nature of our switching concept that acts almost exclusively on the ultrastrongly coupled state in the gap region of the resonator. The decreased transmission observed above $1.6 \mathrm{THz}$ can be attributed to a redshift of the dipolar cavity mode (see Methods and Extended Data Fig. 1). Similarly, the polariton resonances of the 6-QW structure (Fig. 2c) are fully suppressed after photoexcitation (Fig. 2d). 

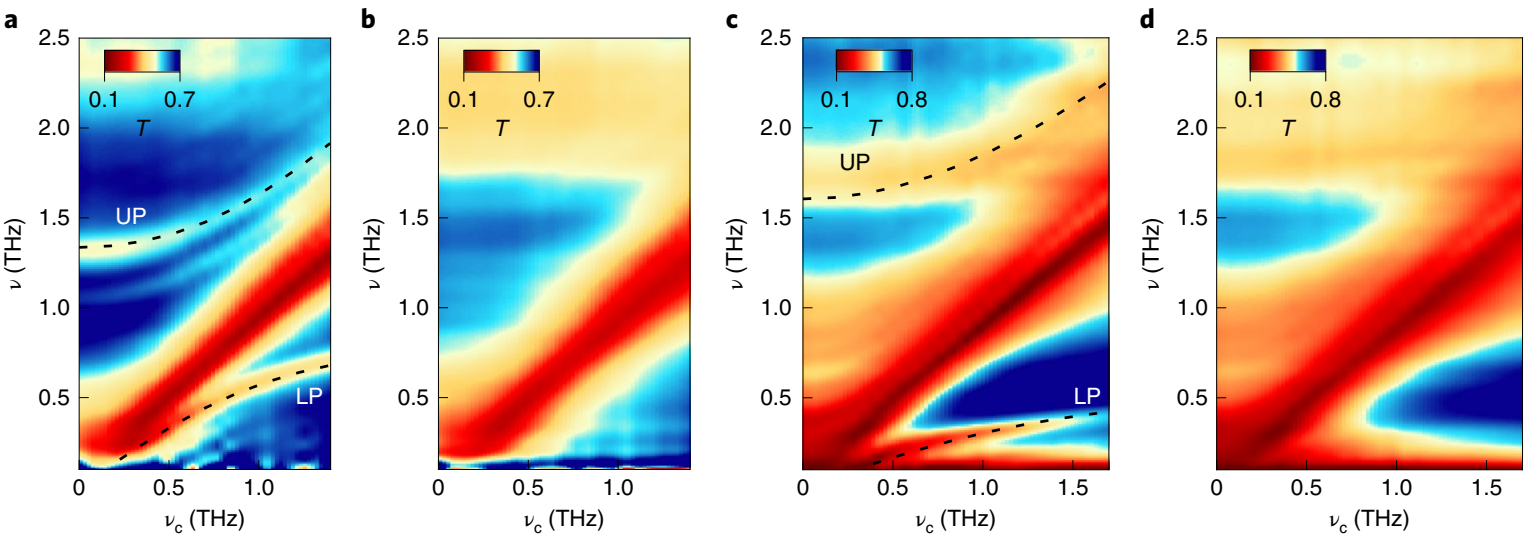

Fig. 2 | Steady-state transmission $T$ through an array of resonators in the deep-strong coupling regime before and after light-matter decoupling. a, Spectra of the 3-QW structure as a function of the CR frequency, $\nu_{c}$. The dashed lines tracing the LP and UP resonances result from a single-mode model of light-matter coupling, and correspond to a normalized coupling strength of $\Omega_{R} / \omega_{1 C}=0.57$. $\mathbf{b}$, After photoexcitation the polariton resonances are absent. c,d, Corresponding transmission spectra of the $6-\mathrm{QW}$ structure with a coupling strength of $\Omega_{\mathrm{R}} / \omega_{\mathrm{LC}}=1.3$ before (c) and after (d) photoexcitation.
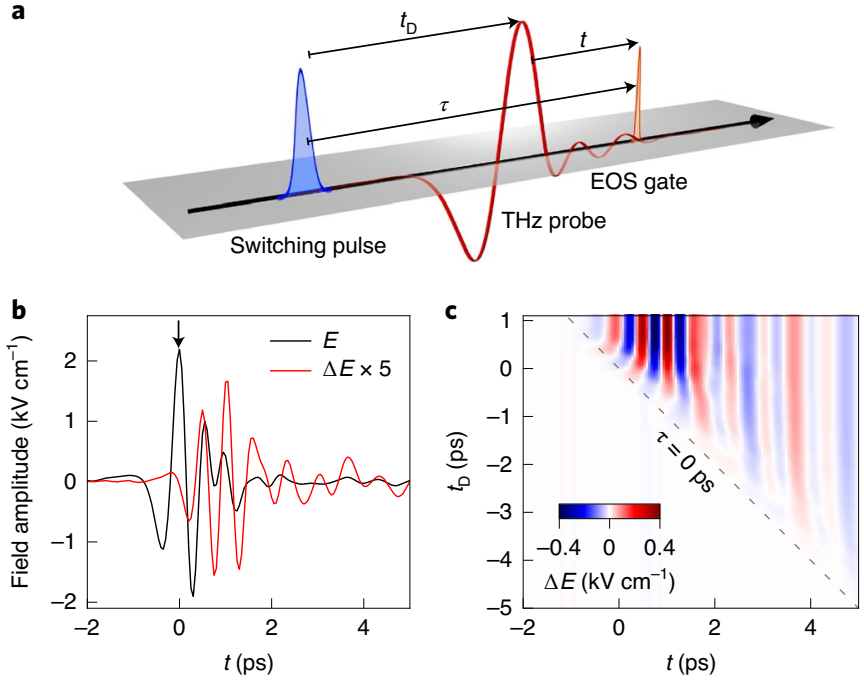

d

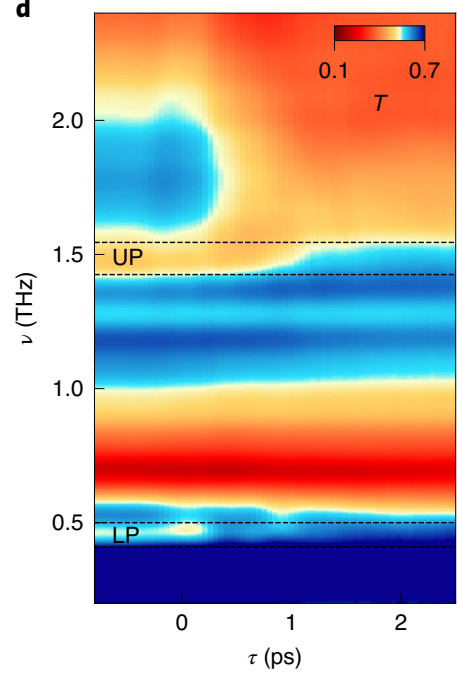

Fig. 3 | Non-adiabatic deactivation dynamics of deep-strong coupling. a, Schematic of excitation. The switching pulse (blue pulse) is shifted with respect to the THz transient (red waveform) by a delay time $t_{D}$, and electro-optic sampling (EOS) is performed at a delay time $t$ (orange pulse). $\mathbf{b}$, Transmitted $\mathrm{THz}$ transient before excitation (black curve) and pump-probe signal $\Delta E$ for $t_{\mathrm{D}}=0 \mathrm{ps}$ (red curve; the temporal position of the maximum of the switching pulse is indicated by the arrow). c, $\Delta E$ as a function of the EOS delay time, $t$, and the pump-probe delay time, $t_{D}$. The grey diagonal line ( $t=-t_{D}$ ) indicates a constant delay $\tau=t+t_{\mathrm{D}}=0$ ps (see $\mathbf{a}$ ). $\mathbf{d}$, Transmission spectra obtained by Fourier transformation of the data in $\mathbf{c}$ along lines of constant $\tau$. The dashed lines indicate the FWHM of the polariton resonances centred at a frequency of $0.48 \mathrm{THz}$ and $1.49 \mathrm{THz}$.

These data hallmark the optically induced collapse of deep-strong light-matter coupling.

\section{Subcycle deactivation dynamics}

Going beyond these quasi-steady-state settings, we explore the subcycle dynamics of light-matter decoupling by measuring the instantaneous $\mathrm{THz}$ response during switching. To this end, the $\mathrm{THz}$ pulse and the switching pulse are shifted with respect to each other by a delay time, $t_{\mathrm{D}}$, and the electro-optic detection is performed at a delay time $t$ relative to the $\mathrm{THz}$ pulse (Fig. $3 \mathrm{a}$ and also see Methods). For photoexcitation at the $\mathrm{THz}$ field crest $\left(t_{\mathrm{D}}=0 \mathrm{ps}\right)$, a strong pump-induced change, $\Delta E$ (Fig. $3 \mathrm{~b}$, red curve), of the transmitted THz field, $E$ (Fig. 3b, black curve), emerges. While the amplitude of $\Delta E$ remains small within the first oscillation half-cycle after switching, it becomes comparable to $E$ during the trailing oscillations
( $t>0.5 \mathrm{ps}$ ), owing to a complete suppression of the narrowband polariton resonances. Figure $3 \mathrm{c}$ depicts a systematic scan of $\Delta E$ as a function of $t_{\mathrm{D}}$. Along the downward diagonal, $t=-t_{\mathrm{D}}$, the switching pulse abruptly disrupts light-matter exchange in various phases of the polariton oscillations.

We will show next that the complex temporal structure displayed in Fig. 3c is characteristic of an abrupt, subcycle collapse of $\Omega_{\mathrm{R}}$ and that this extreme limit manifests in modulations of the response function, on timescales much faster even than the oscillation period of the coupled modes themselves. To extract a well-defined transmission function of the non-equilibrium system, we transformed the transmission data into a different time frame in which $\tau=$ $t+t_{\mathrm{D}}$ represents a constant delay between the switching and the electro-optic gate pulses (Fig. 3 a and also see Methods). Keeping $\tau$ constant (Fig. 3c, dashed line) and performing a Fourier transform 

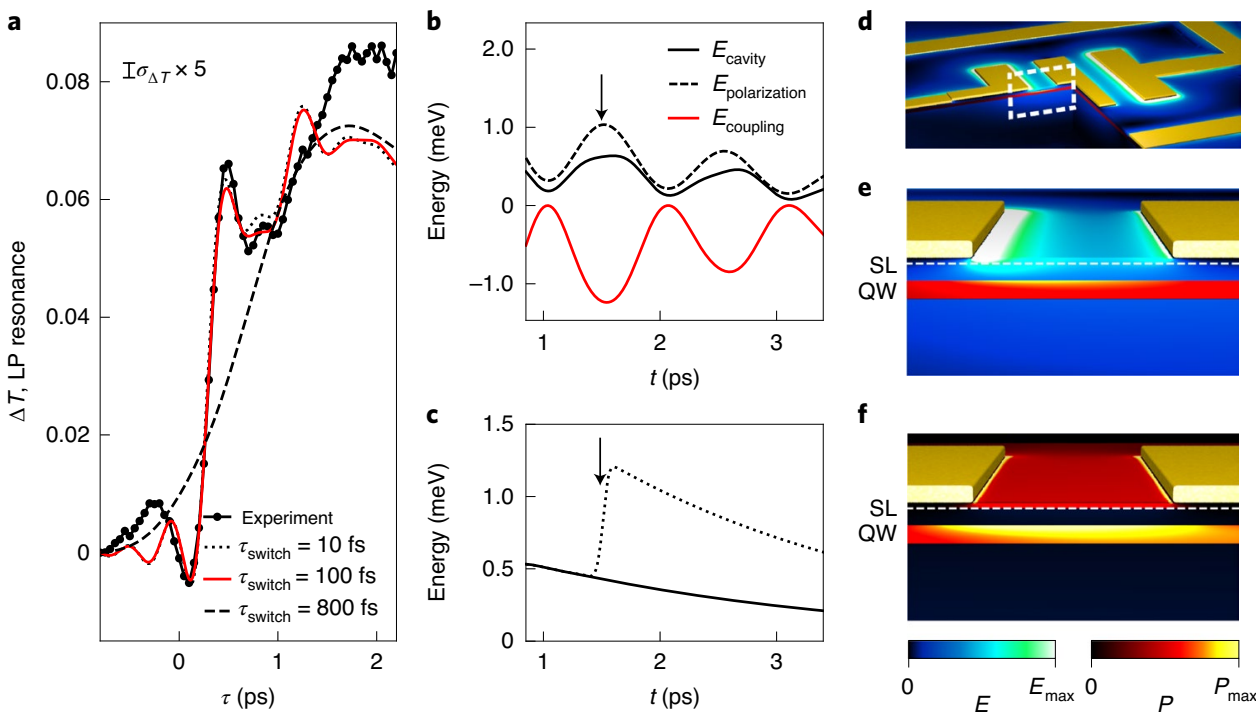

Fig. 4 | Sub-polariton-cycle switching oscillations. a, The differential transmission $\Delta T$ of the LP (solid black curve and circles) extracted within the highlighted spectral range in Fig. 3d shows distinct oscillations on a timescale of less than a single polariton cycle, as confirmed by our calculation for a switching time of $100 \mathrm{fs}$ (red curve). Dotted and dashed curves: calculations of $\Delta T$ for switching times of $10 \mathrm{fs}$, and $800 \mathrm{fs}$, respectively. The error bar indicates the standard deviation of the experimental signal, multiplied by 5, for better visibility. b. Energy of the cavity (solid black curve), polarization energy (dashed black curve) and coupling energy (red curve) when only the LP is excited. The black arrow marks a local extremum of these energies. c, Decay of the total energy without switching (solid black curve) and for switching when the coupling energy is minimized (dotted black curve). d, Cutaway view of the structure visualizing the near fields in the principal planes intersecting in the centre of the resonator, including the electric field, $E$, and the polarization, $P$. e, Close-up of the central gap region highlighted by the dashed rectangle in $\mathbf{d}$, showing the time-averaged fields right before switching. Blue and green colours visualize the electric field, while the polarization of the QW structure is shown in red and yellow colours. The vertical size of the QWs is exaggerated for illustration purposes. The switching layer (SL) is indicated by the dashed line. f, Polarization shortly after the switch-off of light-matter coupling. The SL is strongly polarized. While the electric field of the resonator has collapsed, the electronic polarization is boosted as compared with its time-averaged value before switching.

along $t$ leads to the transmission spectra of Fig. $3 \mathrm{~d}$. The evolution of these spectra as a function of $\tau$ traces the transition from the light-matter-coupled state to the decoupled state. Most remarkably, both polaritons undergo an intriguing oscillatory subcycle change induced by the switching pulse. For a more detailed view, the solid black curve and circles in Fig. 4a show the transmission change, $\Delta T$, at the LP resonance. $\Delta T$ displays a short-lived increase for $\tau$ $<0$ ps and reaches its minimum value near $\tau=0$ ps. Subsequently, $\Delta T$ steeply increases, exhibits an oscillatory feature at $\tau \approx 0.5 \mathrm{ps,}$ and eventually converges to its final value. The dynamics of this sub-polariton-cycle transition entail frequency components exceeding $\nu_{\mathrm{LP}}$ several times. An even more extreme situation prevails for the 6-QW structure (Extended Data Fig. 3), where the transmission at the LP resonance exhibits frequency components exceeding $\nu_{\mathrm{LP}}$ by an order of magnitude. Conversely, switching an empty cavity progresses smoothly (Extended Data Fig. 4).

\section{Subcycle quantum model}

A theoretical analysis can unambiguously explain these high-frequency dynamics by an abrupt deactivation of deep-strong light-matter coupling. To this end, we model the coherent $\mathrm{THz}$ excitation of the polariton states by a mean-field theory of lightmatter interaction including anti-resonant terms and a set of time-dependent parameters that define the cavity modes (see Methods). The effect of the subcycle deactivation of the resonator's LC mode and the resulting collapse of the overlap of light and matter fields are implemented by a single, time-dependent vacuum Rabi frequency, which is reduced to zero in a time interval set by the duration of our switching pulse of $70 \mathrm{fs}$ (see Methods). The calculations trace the dynamics well (Fig. $4 \mathrm{a}$, red curve), reproducing the initial reduction of the transmission, the subsequent steep rise and the transient reduction between $\tau=0.5 \mathrm{ps}$ and $1.1 \mathrm{ps}$. Deviations for $\tau>1.5$ ps are likely to be caused by the dynamics of higher resonator modes not accounted for in our model. Importantly, the theory shows that the oscillations only occur for sufficiently fast switching, whereas long control pulses average out the energy oscillations and lead to a more adiabatic transition (Fig. 4a, black dashed curve). A quantitative match with the experimental data is obtained for switching times between $\tau_{\text {switch }}=10 \mathrm{fs}$ and $100 \mathrm{fs}$ (Fig. $4 \mathrm{a}$ and also see Methods). This upper bound of $\tau_{\text {switch }}$ corresponds to a fraction of $\tau_{\text {switch }} / T_{\mathrm{LP}}=0.02(0.05)$ for our 6-QW (3-QW) structure, where $T_{\mathrm{LP}}=\nu_{\mathrm{LP}}^{-1}$ is the oscillation period of the LP.

These oscillations reflect the internal dynamics of the system in the deep-strong coupling regime. Initially, the $\mathrm{THz}$ pulse prepares a coherent polarization at both polariton frequencies and their counter-propagating wave components. The corresponding anti-resonant interaction terms lead to a complex periodic redistribution of the energies stored in the cavity, $E_{\text {cavity }}$ the electronic excitation, $E_{\text {polarization }}$, and the coupling mechanism itself, $E_{\text {coupling }}$. When the switching pulse abruptly interrupts this transfer, the subsequent dynamics of the uncoupled cavity and electronic polarization are defined by the corresponding instantaneous field amplitudes at the time of switching. The rapid oscillations of the coupled fields progressing faster than a single polariton oscillation cycle lead to equally rapid oscillations of the response function as a function of the switching time. Conversely, switching a set of uncoupled resonances or coupled resonances within the rotating-wave approximation entails qualitatively different dynamics (see Supplementary Information), which underpins the role of anti-resonant interaction mechanisms in the deep-strong coupling regime.

For an intuitive picture of the subcycle energy exchange, we follow the dynamics of the microscopic fields for the case where the total 
energy injected by the THz pulse resides in the LP. This eigenmode is characterized by an out-of-phase oscillation of cavity and matter fields corresponding to an oscillating negative coupling energy (Fig. 4b, red curve). $E_{\text {coupling }}$ reaches the same magnitude as all other energies of the system (see Supplementary Information) and counterbalances the synchronously oscillating $E_{\text {cavity }}$ (Fig. $4 \mathrm{~b}$, solid black curve) and $E_{\text {polarization }}$ (Fig. 4b, dashed black curve). Without switching, the total energy decays exponentially (Fig. 4c, black curve). Here, the coupled gap region of the resonator (Fig. $4 \mathrm{~d}$, dashed area) is permeated by the electric near-field (Fig. 4e, blue to green colours), which interacts strongly with the electronic polarization in the QWs (red colours). In contrast, if the structure is switched, the switching layer is strongly polarized, and the cavity electric field rapidly collapses (Fig. 4f, black colour). If switching occurs near a local minimum of $E_{\text {coupling }}$ (Fig. 4b, black arrow), the negative energy of the coupling mechanism is removed, leading to a rapid increase of the total energy (Fig. 4c, dotted curve). Subsequently, the uncoupled matter mode continues to oscillate freely with an amplitude surpassing its time-averaged value before switching (Fig. 4f, bright yellow colours). The preparation of this unusual, otherwise inaccessible state is thus a direct result of highly non-adiabatic light-matter decoupling.

In conceptual analogy to this situation of a coherent state prepared by a classical $\mathrm{THz}$ field, future experiments may exploit the same mechanism to promote virtual excitations from the incoherent vacuum ground state to real excitations. In combination with recent cutting-edge quantum spectroscopy ${ }^{31,32}$, this concept could form an excellent table-top laboratory to explore quantum electrodynamical phenomena similar to the Unruh-Hawking radiation of black holes ${ }^{28}$.

\section{Perspectives}

Our concept extends subcycle strong-field control of matter to a new arena without strong driving fields and adds time as a new control parameter for the deep-strong coupling regime. Reshaping light-matter hybrid states faster than a cycle of light revealed a novel class of rapid subcycle oscillations of the optical response function with frequency components exceeding the carrier frequency of light by an order of magnitude. The switch-off of deep-strong coupling may even lead to a transfer of energy out of the coupling mechanism, preparing the system in a state otherwise unattainable by external excitation. Among many exciting future perspectives, our approach may be used to switch tailor-cut response functions of all-optically controlled filters and modulators, at arbitrary speed, and explore non-adiabatic quantum optics. By coupling other select low-energy elementary excitations, such as vibrational modes of molecules or lattice vibrations of Josephson plasmons, one may set out to trigger chemical reactions $s^{13,14}$, optically, without the need for a photocatalyst, and steer ultrafast vacuum-modified transport $\mathrm{t}^{11,12}$ as well as cavity-mediated phase transitions, such as superconductivity ${ }^{24,25}$, on demand-none of which require $\mathrm{THz}$ illumination or direct matter excitation.

\section{Online content}

Any methods, additional references, Nature Research reporting summaries, source data, extended data, supplementary information, acknowledgements, peer review information; details of author contributions and competing interests; and statements of data and code availability are available at https://doi.org/10.1038/ s41566-020-0673-2.

Received: 12 December 2019; Accepted: 29 June 2020;

Published online: 10 August 2020

\section{References}

1. Corkum, P. B. \& Krausz, F. Attosecond science. Nat. Phys. 3, 381-387 (2007).

2. Mücke, O. D., Tritschler, T., Wegener, M., Morgner, U. \& Kärtner, F. X. Signatures of carrier-wave Rabi flopping in GaAs. Phys. Rev. Lett. 87, 057401 (2001).

3. Hohenleutner, M. et al. Real-time observation of interfering crystal electrons in high-harmonic generation. Nature 523, 572-575 (2015).

4. Langer, F. et al. Lightwave-driven quasiparticle collisions on a subcycle timescale. Nature 533, 225-229 (2016).

5. Garg, M. et al. Multi-petahertz electronic metrology. Nature 538, 359-363 (2016).

6. Schultze, M. et al. Attosecond band-gap dynamics in silicon. Science $\mathbf{3 4 6}$, 1348-1352 (2014)

7. Ludwig, M. et al. Sub-femtosecond electron transport in a nanoscale gap. Nat. Phys. 16, 341-345 (2020).

8. Kockum, A. F., Miranowicz, A., De Liberato, S., Savasta, S. \& Nori, F. Ultrastrong coupling between light and matter. Nat. Rev. Phys. 1, $19-40$ (2019).

9. Forn-Díaz, P., Lamata, L., Rico, E., Kono, J. \& Solano, E. Ultrastrong coupling regimes of light-matter interaction. Rev. Mod. Phys. 91, 025005 (2019).

10. Li, X. et al. Vacuum Bloch-Siegert shift in Landau polaritons with ultra-high cooperativity. Nat. Photon. 12, 324-329 (2018).

11. Paravicini-Bagliani, G. L. et al. Magneto-transport controlled by Landau polariton states. Nat. Phys. 15, 186-190 (2019).

12. Orgiu, E. et al. Conductivity in organic semiconductors hybridized with the vacuum field. Nat. Mater. 14, 1123-1129 (2015).

13. Thomas, A. et al. Tilting a ground-state reactivity landscape by vibrational strong coupling. Science 363, 615-619 (2019).

14. Chikkaraddy, R. et al. Single-molecule strong coupling at room temperature in plasmonic nanocavities. Nature 535, 127-130 (2016).

15. Anappara, A. et al. Signatures of the ultrastrong light-matter coupling regime. Phys. Rev. B 79, 201303(R) (2009).

16. Günter, A. et al. Sub-cycle switch-on of ultrastrong light-matter interaction. Nature 458, 178-181 (2009).

17. Todorov, Y. et al. Ultrastrong light-matter coupling regime with polariton dots. Phys. Rev. Lett. 105, 196402 (2010).

18. Scalari, G. et al. Ultrastrong coupling of the cyclotron transition of a $2 \mathrm{D}$ electron gas to a THz metamaterial. Science 335, 1323-1326 (2012).

19. Bayer, A. et al. Terahertz light-matter interaction beyond unity coupling strength. Nano Lett. 17, 6340-6344 (2017).

20. Keller, J. et al. Few-electron ultrastrong light-matter coupling at $300 \mathrm{GHz}$ with nanogap hybrid LC microcavities. Nano Lett. 17, 7410-7415 (2017)

21. Yoshihara, F. et al. Superconducting qubit-oscillator circuit beyond the ultrastrong-coupling regime. Nat. Phys. 13, 44-47 (2017).

22. De Liberato, S. Light-matter decoupling in the deep strong coupling regime: the breakdown of the Purcell effect. Phys. Rev. Lett. 112, 016401 (2014).

23. Ciuti, C., Bastard, G. \& Carusotto, I. Quantum vacuum properties of the intersubband cavity polariton field. Phys. Rev. B 72, 115303 (2005).

24. Schlawin, F., Cavalleri, A. \& Jaksch, D. Cavity-mediated electron-photon superconductivity. Phys. Rev. Lett. 122, 133602 (2019).

25. Thomas, A. et al. Exploring superconductivity under strong coupling with the vacuum electromagnetic field. Preprint at https://arxiv.org/abs/1911.01459v2 (2019)

26. De Liberato, S., Ciuti, C. \& Carusotto, I. Quantum vacuum radiation spectra from a semiconductor microcavity with a time-modulated vacuum Rabi frequency. Phys. Rev. Lett. 98, 103602 (2007).

27. Wilson, C. M. et al. Observation of the dynamical Casimir effect in a superconducting circuit. Nature 479, 376-379 (2011).

28. Hawking, S. W. Black hole explosions? Nature 248, 30-31 (1974).

29. Chen, H.-T. et al. Experimental demonstration of frequency-agile terahertz metamaterials. Nat. Photon. 2, 295-298 (2008).

30. Bühler, J., Allerbeck, J., Fitzky, G., Brida, D. \& Leitenstorfer, A. Terahertz shockwaves generated by a precise subcycle cut of the electric field. Optica 5, 821-824 (2018).

31. Riek, C. et al. Subcycle quantum electrodynamics. Nature 541, 376-379 (2017).

32. Benea-Chelmus, I. C., Settembrini, F. F., Scalari, G. \& Faist, J. Electric field correlation measurements on the electromagnetic vacuum state. Nature $\mathbf{4 1 4}$, 286-289 (2001).

Publisher's note Springer Nature remains neutral with regard to jurisdictional claims in published maps and institutional affiliations.

(C) The Author(s), under exclusive licence to Springer Nature Limited 2020 


\section{Methods}

Sample fabrication. Our symmetrically doped QWs were fabricated by molecular beam epitaxy on a GaAs substrate, and capped by an $\operatorname{In}_{0.5} \mathrm{Ga}_{0.45}$ As layer of a thickness of $100 \mathrm{~nm}$. This InGaAs layer exhibits an energy gap of $0.73 \mathrm{eV}$ and an intentionally defect-rich crystal structure that still exhibits photoconductivity, but does not allow a CR to build up. The InGaAs switching patches of an area of $18 \mu \mathrm{m} \times 22 \mu \mathrm{m}$ were formed by electron-beam lithography and wet-chemical etching. The gold $\mathrm{THz}$ resonators were subsequently fabricated on top, whereby their gaps were aligned with the switching patches. In a final step, we glued the sample onto a sapphire window and removed the GaAs substrate in order to prevent effects from two-photon absorption of the switching pulse in the substrate.

Design of switchable resonators. The design of our switchable structures is based on finite-element frequency-domain calculations in analogy to ref. ${ }^{19}$. The $\mathrm{THz}$ resonators are arranged in quadratic unit cells of a length of $60 \mu \mathrm{m}$. They feature a capacitive gap element in which the electric field of the fundamental LC mode is enhanced by up to a factor of 8 with respect to the far field (Extended Data Fig. 1a). In contrast, the near field of the higher-energy, dipolar mode (DP) is strongly enhanced at the outer perimeter of the resonator (Extended Data Fig. 1b), allowing for selective switching of the LC mode by photodoping of the switching patch underneath the central gap. We applied the Drude model for a charge carrier plasma to calculate the contribution that the photoexcitation adds to the dielectric response of the switching patch. The plasma frequency is given by

$$
\omega_{\mathrm{p}}=\sqrt{\frac{\rho_{\mathrm{switch}} e^{2}}{\epsilon_{0} m_{\mathrm{InGaAs}}}} .
$$

Here $m_{\mathrm{InGaAs}}=0.04 m_{\mathrm{e}}$ denotes the effective mass of conduction-band electrons in InGaAs, $m_{\mathrm{e}}$ is the free-electron mass, $\rho_{\text {switch }}$ is the electron charge carrier density, $\varepsilon_{0}$ is the vacuum permittivity and $e$ is the elementary charge. In the experiment, we chose an optical fluence of $135 \mu \mathrm{J} \mathrm{cm}^{-2}$ for the switching pulse, leading to saturation of the switching effect. We thus assumed a density of photoexcited carriers of $\rho_{\text {switch }}$ $=3.5 \times 10^{18} \mathrm{~cm}^{-3}$, corresponding to the total density of states of InGaAs within the bandwidth of our switching pulse. As a result, we obtained a plasma frequency of $\nu_{\mathrm{p}}=\omega_{\mathrm{p}} / 2 \pi=80 \mathrm{THz}$. The dielectric function of the plasma is then given by

$$
\epsilon_{\mathrm{InGaAs}}(\omega)=\epsilon_{\mathrm{InGaAs}}(\infty)-\frac{\omega_{\mathrm{p}}^{2} \tau_{\mathrm{s}}^{2}}{\omega^{2} \tau_{\mathrm{s}}^{2}+i \omega \tau_{\mathrm{s}}},
$$

where we chose a scattering time of $\tau_{\mathrm{s}}=1 \times 10^{-13} \mathrm{~s}$ and a static dielectric constant of $\epsilon_{\text {InGaAs }}(\infty)=13.7$. We verified our switching concept by calculating the equilibrium transmission of the switched resonator based on this dielectric response. While the strong screening of the electric field generally reshapes both modes, the impact on the LC mode is much stronger as compared with the DP mode (Extended Data Fig. 1c,d). Correspondingly, the calculated transmission spectra reveal that the LC mode is completely quenched, while the DP mode experiences a redshift of $0.25 \mathrm{THz}$ (Extended Data Fig. 1e).

We calculated the response of our coupled structures by extending our model with the gyrotropic response of Landau-quantized electrons in the QWs analogously to the procedure detailed in ref. ${ }^{19}$. The calculated equilibrium spectra (see Supplementary Information) are in agreement with the experimental data and show a distinct LP resonance. Activating the switching patch, the calculations predict a collapse of the light-matter coupling of the fundamental LC mode, as the LP branch disappears completely (see Supplementary Information). Other coupled resonances at frequencies above $\nu_{c}$ experience a slight spectral shift imposed by the redshift of the DP mode, but remain mostly unaffected otherwise.

Calculation of the coupling strength. For coupling strengths approaching or entering the deep-strong coupling regime, the influence of cavity modes at energies above the resonantly coupled LC mode changes the overall spectrum of the coupled system. In our THz resonators, the frequency of the DP mode was approximately twice as large as the frequency of the LC mode. As a result, we observed only a small influence of multi-mode coupling that manifests as additional spectral features in the vicinity of the UP branch (Fig. 2a,c). However, the LP branch remains clearly isolated. We thus determined the coupling strength by applying Hopfield's model in the usual single-mode approximation ${ }^{33}$, taking into account the frequencies of the LP and the CR, as a function of $\nu_{\mathrm{c}}$.

Experimental set-up. The layout of our subcycle spectroscopic set-up is detailed in Extended Data Fig. 2. Femtosecond near-infrared pulses (centre wavelength, $807 \mathrm{~nm}$; pulse energy, $5.5 \mathrm{~mJ}$; pulse duration, $33 \mathrm{fs}$ ) from a Ti:sapphire amplifier laser (repetition rate, $3 \mathrm{kHz}$ ) were split by a beam splitter into two branches, which drive an optical parametric amplifier, and $\mathrm{THz}$ generation and detection, respectively. The $\mathrm{THz}$ generation beam was sent across a delay line introducing the delay time $t_{\mathrm{D}}$, and subsequently focused into a $\langle 110\rangle$-cut ZnTe crystal of a thickness of $1 \mathrm{~mm}$ to generate single-cycle $\mathrm{THz}$ pulses by optical rectification. The $\mathrm{THz}$ pulses were focused through the sample before the transmitted waveforms were recollimated and focused onto a ZnTe detector crystal using off-axis parabolic mirrors. A small fraction of the near-infrared power was split off after the first delay line, passed through a second delay line to introduce the delay time $t$, and used for electro-optic detection of the THz pulses in the detector crystal using polarization optics and a pair of balanced photodiodes. The optical parametric amplifier generated the near-infrared switching pulses of a duration of $70 \mathrm{fs}$ (full-width at half-maximum (FWHM)) and a centre photon energy of $1.03 \mathrm{eV}$. These pulses were focused onto the sample with a spot diameter of $4 \mathrm{~mm}$ (FWHM) for homogeneous excitation. Two mechanical choppers modulated the $\mathrm{THz}$ and switching pulses, allowing for differential detection of the pump-induced change of the transmitted THz electric field, $\Delta E\left(t, t_{\mathrm{D}}\right)$. The linear conversion between $\Delta E(t$, $\left.t_{\mathrm{D}}\right)$ and $\Delta \tilde{E}\left(t, \tau=t+t_{\mathrm{D}}\right)$ was performed numerically ${ }^{34}$. The sample was kept at a temperature of $10 \mathrm{~K}$ in a magneto-cryostat with a large numerical aperture.

Light-matter decoupling dynamics of the 6-QW structure. Our most strongly coupled structure was based on a 6-QW structure with a charge carrier concentration of $1.75 \times 10^{12} \mathrm{~cm}^{-2}$ per QW, and a THz resonator with capacitive gaps located at the outer perimeter (Extended Data Fig. 3a,b). The resulting coupling strength was $\Omega_{\mathrm{R}} / \omega_{\mathrm{LC}}=1.3$. The light-matter decoupling dynamics (Extended Data Fig. $3 \mathrm{c}-\mathrm{e}$ ) displayed qualitatively similar yet more extreme sub-polariton-cycle oscillations as compared with the 3-QW structure (Figs. 3 and 4). In particular, the dynamics included frequencies exceeding the frequency of the LP mode by more than an order of magnitude (Extended Data Fig. 3f).

Switching dynamics of an uncoupled THz resonator. As a control experiment, we investigated the switching dynamics of an uncoupled $\mathrm{THz}$ resonator identical to the 3-QW structure, yet without QWs. The pump-probe THz field as a function of delay time, $t$, and pump-probe delay time, $t_{\mathrm{D}}$ (Extended Data Fig. $4 \mathrm{a}$ ) was obtained by performing the same subcycle switching measurement as for the coupled structures. The resulting time-dependent transmission spectra (Extended Data Fig. 4b) demonstrate the spectral selectivity of the switching concept that affects mostly the narrowband range of the fundamental LC mode of the resonator at $0.8 \mathrm{THz}$, and the more broadband DP mode at $1.8 \mathrm{THz}$. After an initial reduction of the transmission, the switching dynamics of the fundamental mode progressed monotonically (Extended Data Fig. 4c, black curve), leading to an overall increase of the transmission by 0.18 after switching. By contrast, the transmission extracted at a frequency of $2.1 \mathrm{THz}$, for reference, was left mostly unchanged (Extended Data Fig. 4c, red curve)

Quantum model of non-adiabatic deactivation dynamics of deep-strong coupling. We theoretically investigated the decoupling dynamics of our structures by a theory developed specifically for our setting, which treats light-matter interaction in the deep-strong coupling regime beyond the rotating-wave approximation, on subcycle timescales. Light-matter coupling was implemented between the LC mode and the Landau-quantized electrons with a vacuum Rab frequency $\Omega_{\mathrm{R}}$. While light-matter coupling of the DP mode was neglected, its influence on the transmission and its frequency shift during switching are included in the theory. The Hamiltonian

$$
\hat{H}=\hat{H}_{\text {cavity }}+\hat{H}_{\text {Landau }}+\hat{H}_{\text {int }}+\hat{H}_{\text {dia }}+\hat{H}_{\text {ext }}
$$

accounts for the cavity modes via

$$
\hat{H}_{\text {cavity }}=\sum_{j} \hbar \omega_{j} \hat{a}_{j}^{\dagger} \hat{a}_{j}
$$

where $\hat{a}_{j}$ is the bosonic annihilation operator of the LC $(j=\mathrm{LC})$ and $\mathrm{DP}(j=\mathrm{DP})$ modes. Excitations of the matter mode are implemented assuming a large number of Landau electrons and a weak THz excitation, whereby $\hat{b}$ is the corresponding bosonic annihilation operator and $\omega_{\mathrm{c}}$ is the cyclotron resonance frequency

$$
\hat{H}_{\text {Landau }}=\hbar \omega_{\mathrm{c}} \hat{b}^{\dagger} \hat{b}
$$

Light-matter interaction is described by the operator

$$
\hat{H}_{\mathrm{int}}=\hbar \Omega_{\mathrm{R}}\left(\hat{a}_{\mathrm{LC}}+\hat{a}_{\mathrm{LC}}^{\dagger}\right)\left(\hat{b}+\hat{b}^{\dagger}\right)
$$

and contains both resonant terms as well as anti-resonant contributions proportional to $\hat{a}_{\mathrm{LC}} \hat{b}$ and $\hat{a}_{\mathrm{LC}}^{\dagger} \hat{b}^{\dagger}$, which take the dynamics in the deep-strong coupling regime into account. Furthermore, the cavity experiences a blueshift by diamagnetic interactions

$$
\hat{H}_{\text {dia }}=\hbar D\left(\hat{a}_{\mathrm{LC}}+\hat{a}_{\mathrm{LC}}^{\dagger}\right)^{2}
$$

where $D=\Omega_{\mathrm{R}}^{2} / \omega_{\mathrm{c}}$. Finally, the coupling to the external THz field, $E_{\text {ext }}(t)$, is contained in $\hat{H}_{\text {ext. }}$.

We derive the subcycle dynamics of the cavity and matter fields by applying the Heisenberg equation of motion and performing a mean-field treatment, $\alpha_{j}=\hat{a}_{j}$ and $\beta=\hat{b}$. The resulting equations of motion read

$$
\begin{aligned}
\frac{\mathrm{d}}{\mathrm{d} t} \alpha_{\mathrm{LC}}= & -i \omega_{\mathrm{LC}} \alpha_{\mathrm{LC}}-\gamma_{\mathrm{LC}} \alpha_{\mathrm{LC}}-i \Omega_{\mathrm{R}}\left[\beta(t)+\beta^{*}(t)\right]-2 i D\left[\alpha_{\mathrm{LC}}(t)+\alpha_{\mathrm{LC}}^{*}(t)\right] \\
& +\kappa_{\mathrm{LC}} E_{\mathrm{ext}}(t)
\end{aligned}
$$




$$
\begin{aligned}
& \frac{\mathrm{d}}{\mathrm{d} t} \alpha_{\mathrm{DP}}=-i \omega_{\mathrm{DP}} \alpha_{\mathrm{DP}}-\gamma_{\mathrm{DP}} \alpha_{\mathrm{DP}}+\kappa_{\mathrm{DP}} E_{\mathrm{ext}}(t), \\
& \frac{\mathrm{d}}{\mathrm{d} t} \beta=-i \omega_{\mathrm{c}} \beta-\gamma_{\mathrm{c}} \beta-i \Omega_{\mathrm{R}}\left[\alpha_{\mathrm{LC}}(t)+\alpha_{\mathrm{LC}}^{*}(t)\right],
\end{aligned}
$$

where we introduced damping terms $\gamma_{j}$ and $\gamma_{c}$ for the cavity and matter modes, respectively, and scaled each cavity mode's coupling to the external field by an individual coupling parameter $\kappa_{j}$.

This set of coupled differential equations models the response of our structure for fixed parameters of the cavity and matter modes. Next, we implemented the decoupling mechanism by a time-dependent vacuum Rabi frequency $\Omega_{\mathrm{R}}(t+$ $t_{\mathrm{D}}$ ), which transitions from its equilibrium value, $\Omega_{\mathrm{R}}^{\mathrm{i}}$, to its value after switching, $\Omega_{\mathrm{R}}^{\mathrm{f}}=0$, whereby its instantaneous change is proportional to the rate of generation of photoexcited carriers. For the switching pulse, we used a Gaussian temporal shape with a FWHM of $\tau_{\text {switch }}$ and obtained

$$
\Omega_{\mathrm{R}}\left(t+t_{\mathrm{D}}\right)=\Omega_{\mathrm{R}}^{\mathrm{i}}+\frac{\left(\Omega_{\mathrm{R}}^{\mathrm{f}}-\Omega_{\mathrm{R}}^{\mathrm{i}}\right)}{2}\left(1+\frac{2}{\sqrt{\pi}} \int_{0}^{t+t_{\mathrm{D}}} \mathrm{e}^{-\left(\frac{\sqrt{4 \ln 2}}{\tau_{\text {switch }}}\left(t_{\mathrm{D}}^{\prime}\right)\right)^{2}} \mathrm{~d} t_{\mathrm{D}}^{\prime}\right) .
$$

We furthermore introduced dynamical dampings, $\gamma_{j}\left(t+t_{\mathrm{D}}\right)$, and coupling parameters, $\kappa_{j}\left(t+t_{\mathrm{D}}\right)$, for the LC and DP modes. For the LC cavity mode, we reduced the coupling to the external field by $50 \%$. We implemented the frequency shift of the DP mode by a corresponding time-dependent value $\omega_{\mathrm{DP}} / 2 \pi$ that is shifted from $2.2 \mathrm{THz}$ to $1.8 \mathrm{THz}$, while its far-field coupling is increased by $45 \%$. Solving for these dynamics, we obtained the electric field $E\left(t, t_{\mathrm{D}}\right)$ as a function of $t_{\mathrm{D}}$, and the corresponding transmission $\Delta T(\nu, \tau)$ (ref. $\left.{ }^{34}\right)$ (Fig. 4).

Estimation of the light-matter decoupling time. Our light-matter decoupling mechanism is based on the screening of the electric near-field of the fundamental $\mathrm{LC}$ mode of the $\mathrm{THz}$ resonators by a charge carrier plasma created by femtosecond excitation with near-infrared optical pulses. The switching pulses of a duration of $70 \mathrm{fs}$ (FWHM) created a carrier density of $\rho_{\text {switch }}=3.5 \times 10^{18} \mathrm{~cm}^{-3}$ corresponding to an electron plasma frequency of $\nu_{\mathrm{p}}=80 \mathrm{THz}$. The time for the build-up of screening ${ }^{35}, t_{\mathrm{c}}=\nu_{\mathrm{p}}^{-1}=12.5 \mathrm{fs}$, led us to expect that the near field is cut off within approximately the duration of the switching pulse.

We verified this estimate by a series of calculations based on our dynamical model, in which we systematically varied $\tau_{\text {switch }}$ for our 3-QW structure. The calculations showed that the amplitude of the oscillatory dynamics matched the experimental data only for $\tau_{\text {switch }}<100 \mathrm{fs}$, which we thus identified as an upper limit of the light-matter decoupling time, compatible with the estimate based on the plasma frequency. Larger values of $\tau_{\text {switch }}$, on the contrary, led to a reduction of the oscillation amplitude up to a completely monotonic increase of $T$ (Fig. 4a, black dashed curve).

\section{Data availability}

The data that support the plots within this paper and other findings of this study are available from the corresponding authors upon reasonable request.

\section{References}

33. Hopfield, J. J. Theory of the contribution of excitons to the complex dielectric constant of crystals. Phys. Rev. 112, 1555-1567 (1958).

34. Kindt, J. T. \& Schmuttenmaer, C. A. Theory for determination of the low-frequency time-dependent response function in liquids using time-resolved terahertz pulse spectroscopy. J. Chem. Phys. 110, 8589-8596 (1999).

35. Huber, R. et al. How many-particle interactions develop after ultrafast excitation of an electron-hole plasma. Nature 414, 286-289 (2001).

\section{Acknowledgements}

The work in Regensburg was supported by the Deutsche Forschungsgemeinschaft (DFG) through grant numbers LA 3307/1-2, BO 3140/3-2 and HU 1598/2 as well as by the European Research Council (ERC) under the European Union's Horizon 2020 research and innovation programme by grant agreement number 305003, Ultrafast quantum physics on the sub-cycle time scale (QUANTUMsubCYCLE), and through Future and Emerging Technologies (FET) grant number 737017, Mid- and far-IR optoelectronic devices based on Bose-Einstein condensation (MIR-BOSE).

\section{Author contributions}

C.L., M.H., V.Z., D.B. and R.H. designed and implemented the structures. M.H., J.M. and C.L. carried out the experiment with support from R.H. The theoretical modelling was carried out by C.L., M.H., J.M., R.H. and C.C. C.L., D.B. and R.H. supervised the study. All authors analysed the data, discussed the results and contributed to the writing of the manuscript.

\section{Competing interests}

The authors declare no competing interests.

\section{Additional information}

Extended data is available for this paper at https://doi.org/10.1038/s41566-020-0673-2. Supplementary information is available for this paper at https://doi.org/10.1038/ s41566-020-0673-2.

Correspondence and requests for materials should be addressed to R.H. or C.L. Reprints and permissions information is available at www.nature.com/reprints. 

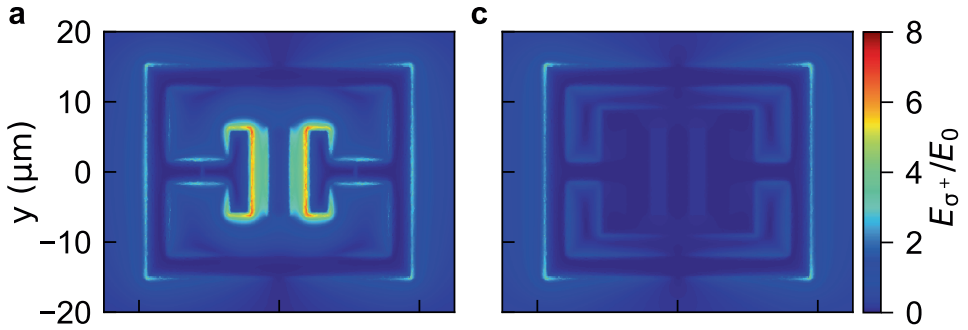

b

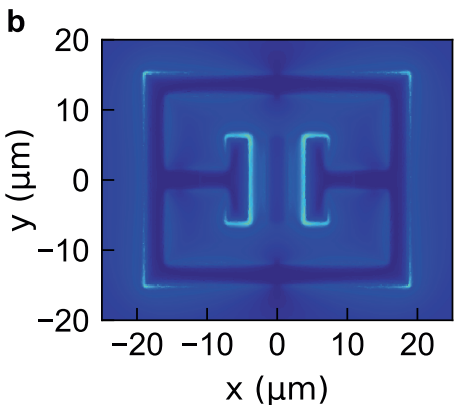

d

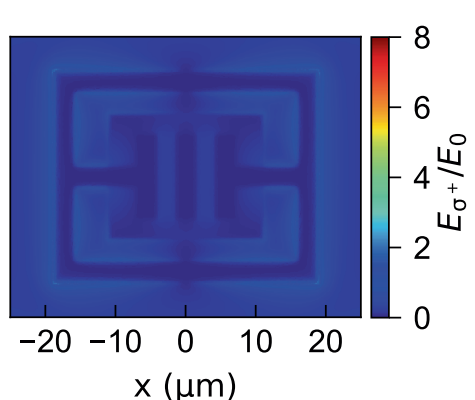

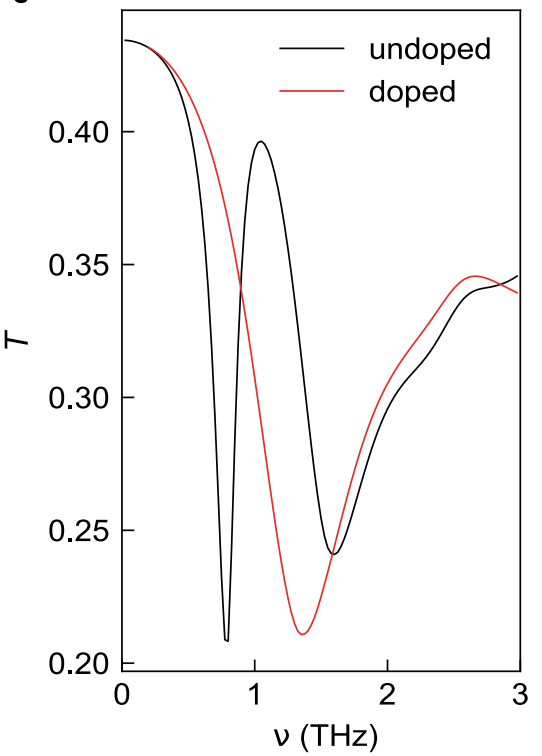

Extended Data Fig. 1 | Calculated spatial and spectral characteristics of the resonator structure. a, Enhancement of the amplitude of the right-circularly polarized near-field component, $E_{\sigma}^{+}$, of the fundamental LC mode, relative to the far-field amplitude, $E_{0}$, at a depth of z $=-200 \mathrm{~nm}$, for an undoped switching layer (see Fig. 1). b, Corresponding near-field enhancement of the dipolar mode. c, Near-field enhancement with a doped switching layer, for the LC mode, and d for the dipolar mode. e, Transmission spectra for a doped and an undoped switching patch, respectively. 


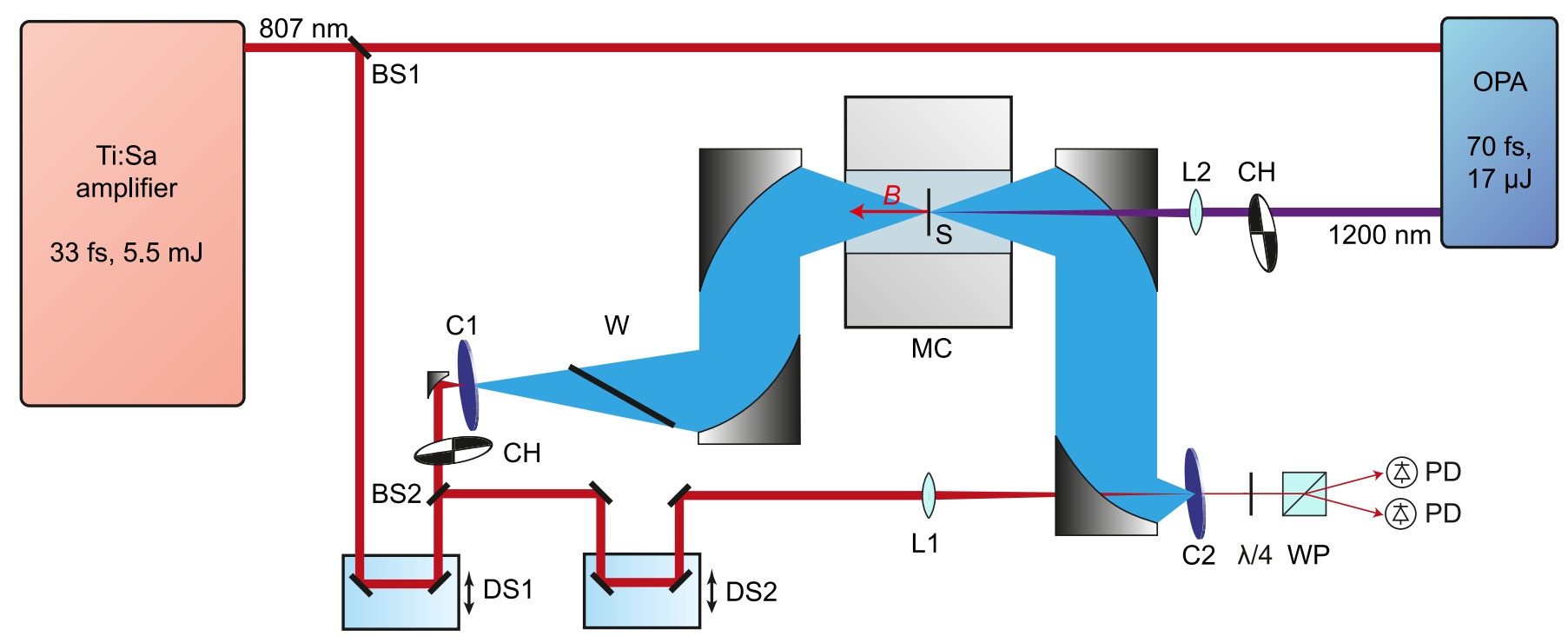

Extended Data Fig. 2 | Experimental setup. The near-infrared (NIR) pulse train (centre wavelength, $807 \mathrm{~nm}$; pulse energy, $5.5 \mathrm{~mJ}$, pulse duration, $33 \mathrm{fs}$ ) generated in a titanium-sapphire amplifier (repetition rate, $3 \mathrm{kHz}$ ) is split by a beam splitter (BS1) into a first branch driving an optical parametric amplifier (OPA) and a second branch for THz generation and detection. A delay stage (DS1) controls the common delay, $t_{\mathrm{D}}$ of $\mathrm{THz}$ and electro-optic sampling pulses. A second beam splitter (BS2) picks off the gate pulse which is delayed by a second stage (DS2) controlling the electro-optic sampling delay, $t$. The gate is focused through the detection crystal (C2) by a lens (L1), after which its polarisation state is analysed by corresponding optics ( $\lambda / 4$, quarter wave plate; WP, Wollaston prism; PD, photodiodes). The NIR power transmitted through BS2 is focused onto the THz generation crystal (C1) by an off-axis parabolic mirror. A wafer (W) transmits only the generated THz waveform. Off- axis parabolic mirrors recollimate and focus the pulses through the sample (S), which is located in the magnet cryostat (MC) and kept at a temperature of $10 \mathrm{~K}$. Here, a magnetic bias $(B)$ of up to $5.5 \mathrm{~T}$ is applied perpendicular to the sample surface. The transmitted THz waveform is recollimated and focused onto the detector crystal, collinearly with the gate pulse. The NIR signal output from the OPA (centre wavelength, $1200 \mathrm{~nm}$; pulse energy, $17 \mu \mathrm{J}$, pulse duration, $70 \mathrm{fs}$ ) is focused by a lens (L2) onto the focal point of the THz pulses on the sample. Mechanical choppers $(\mathrm{CH})$ are placed in the beam paths of $\mathrm{THz}$ generation and switching pulses to enable differential detection. 


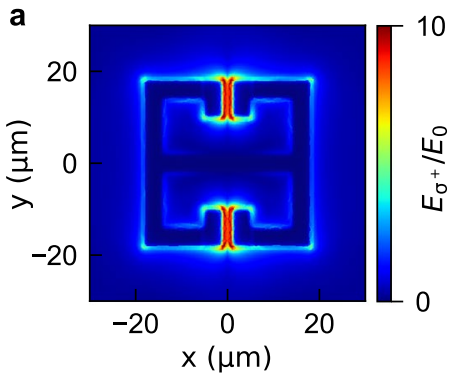

b

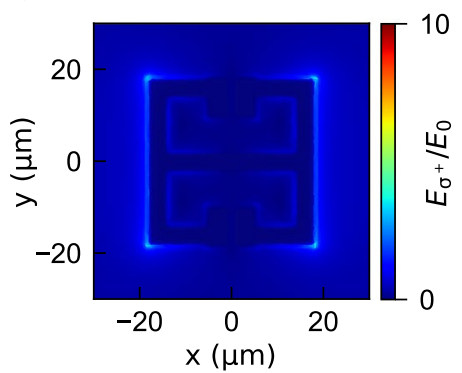

C

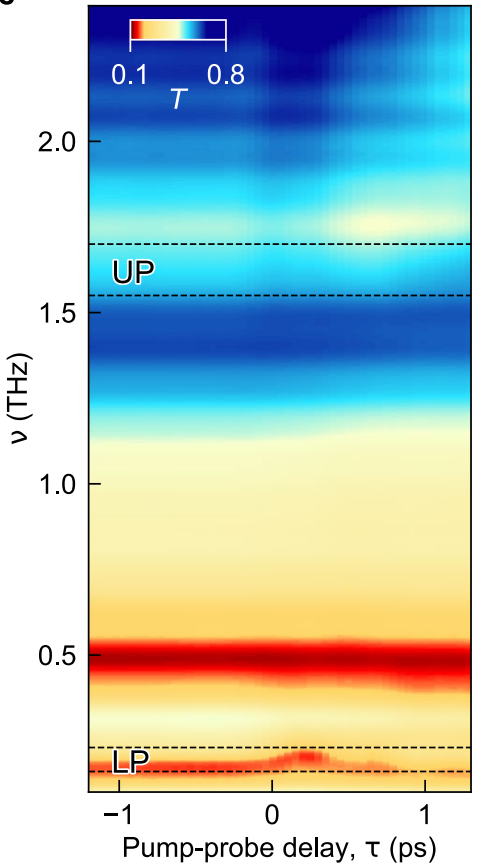

d

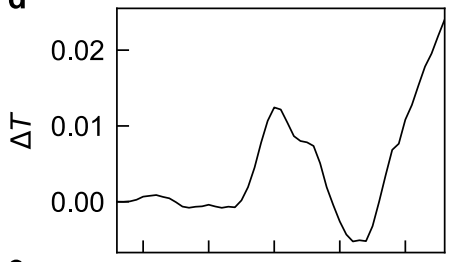

e
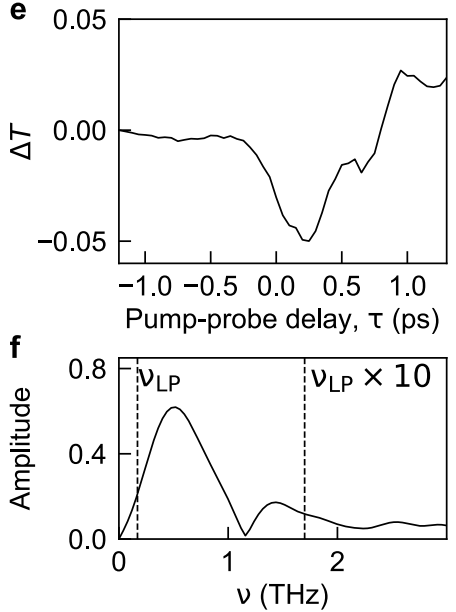

Extended Data Fig. 3 | Subcycle switching dynamics of the 6-QW structure with $\Omega_{\mathrm{R}} / \omega_{\mathrm{LC}}=1.3$. a, Enhancement of the right-circularly polarized near-field amplitude, $E_{\sigma}{ }^{+}$, of the fundamental LC mode relative to the far-field amplitude, $E_{0}$, at a depth of $\mathrm{z}=-200 \mathrm{~nm}$, for an unexcited switching layer. $\mathbf{b}$,

Corresponding near-field enhancement with a photoexcited switching layer. $\mathbf{c}$, Transmission spectra of the 6-QW structure as a function of pump-probe delay time, $\tau$, at the anti-crossing point. The dashed lines mark the frequency windows of the lower and upper polariton resonances at $0.17 \mathrm{THz}$ and $1.62 \mathrm{THz}$, respectively. The transmission exhibits oscillatory dynamics at the frequency of $\mathbf{d}$, the UP, and $\mathbf{e}$, the LP, caused by the non-adiabatic switch-off. $\mathbf{f}$, Spectrum of the switching dynamics of the LP of panel e. Vertical dashed lines indicate the resonance frequency of the LP, $\nu_{L P}$ and $10 \times v_{L P}$, for reference. 
a

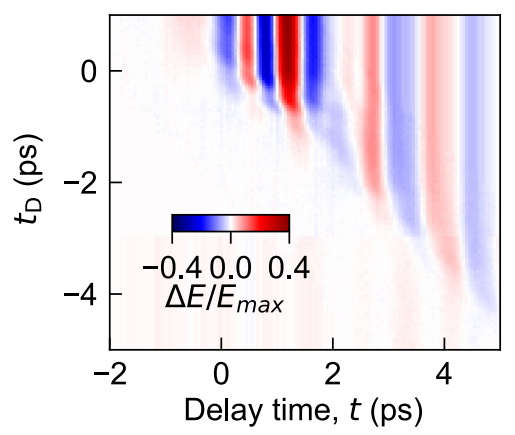

b

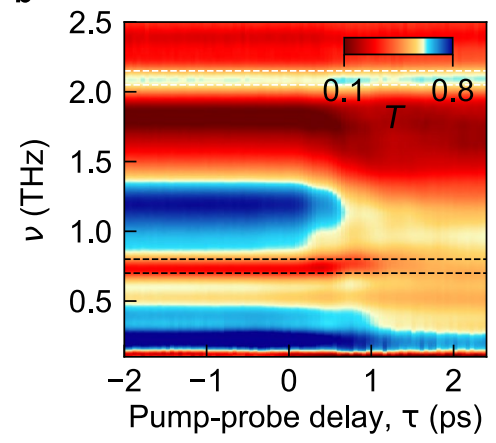

C

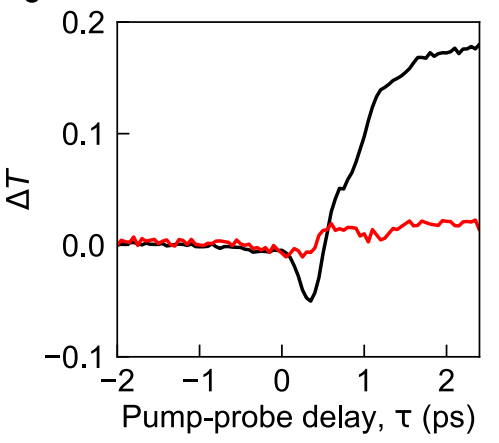

Extended Data Fig. 4 | Switching dynamics of an uncoupled THz resonator. a, Pump-probe THz signal during femtosecond photoexcitation. The charge carrier concentration resulting from femtosecond photoexcitation is $3.5 \times 10^{18} \mathrm{~cm}^{-3}$. b. Corresponding transmission spectra, in the $\tau$-frame. $\mathbf{c}$, Black curve: Differential transmission of the LC mode extracted within the frequency window outlined in panel $\mathbf{b}$ by dashed black lines. Red curve: Differential transmission extracted within the frequency window outlined in panel $\mathbf{b}$ by dashed white lines, for reference. 